\section{$\underset{\text { \& migrations }}{\text { hommes }}$}

\section{Hommes \& migrations}

Revue française de référence sur les dynamiques

migratoires

$1312 \mid 2015$

Diasporas iraniennes

\title{
Les touristes iraniens à Istanbul
}

Des « fourmis voyageuses » de la mondialisation

\section{Mina Saïdi-Sharouz}

\section{Q OpenEdition}

1 Journals

\section{Édition électronique}

URL : http://journals.openedition.org/hommesmigrations/3498

DOI : 10.4000/hommesmigrations.3498

ISSN : 2262-3353

Éditeur

Musée national de l'histoire de l'immigration

Édition imprimée

Date de publication : 1 octobre 2015

Pagination : 81-89

ISBN : 978-2-919040-33-9

ISSN : 1142-852X

Référence électronique

Mina Saïdi-Sharouz, « Les touristes iraniens à Istanbul », Hommes \& migrations [En ligne], 1312 | 2015, mis en ligne le 01 octobre 2018, consulté le 20 avril 2019. URL : http://journals.openedition.org/

hommesmigrations/3498; DOI : 10.4000/hommesmigrations.3498 


\section{LES TOURISTES IRANIENS À ISTANBUL}

\section{LES "FOURMIS VOYAGEUSES" DE LA MONDIALISATION}

par MINA SAÏDI-SHAROUZ, docteure en géographie urbaine, École nationale supérieure d'architecture de Paris-La Villette, laboratoire Mosaïques/LAVUE.

La Turquie, Istanbul notamment, est devenue

une destination de rêve pour un grand nombre d'Iraniens.

La vie moderne, le luxe, la liberté des mœurs, etc., autant de représentations réelles ou fantasmées qui font l'attractivité de la plus grande métropole du pays et poussent de plus en plus d'Iraniens à s'y rendre. Ville de loisirs qui offre aux Iraniens de se retrouver hors des frontières d'Iran, Istanbul constitue également un carrefour commercial en plein essor pour les biens de consommation et l'immobilier.

Selon les chiffres publiés par les compagnies aériennes, plus d'un million d'Iraniens se rendent chaque année à Istanbul. Ce chiffre est en hausse selon l'agence de presse officielle de la République islamique d'Iran ${ }^{1}$. En 2015, lors des deux premiers mois de l'année, 2,5 millions de voyages ont été effectués en Turquie, dont 1,2 millions d'Iraniens. Ces voyages sont d'autant plus facilités qu'ils ne nécessitent pas de formalités douanières importantes et que la Turquie est un des rares pays qui n'exige pas de visa pour les Iraniens.

En dehors des villes côtières telles qu'Antalya, Analya ou Bodrum, Istanbul, étape incontournable, reste la destination la plus fréquentée par les tou- ristes iraniens. Cette mégapole, entre l'Orient et l'Occident, offre de loin l'image d'une ville d'accueil cosmopolite et multiconfessionnelle. Une image qui renforce l'attraction des touristes étrangers. Istanbul soigne ses multiples facettes, de la plus traditionnelle à la plus moderne, et ses ambiances variées conviennent à tous les goûts. Les Iraniens s'y plaisent particulièrement car ils peuvent se sentir chez eux tout en trouvant un mode de vie différent, proche de la vie occidentale ${ }^{2}$.

En se basant sur l'expérience de nos fréquents voyages entre Téhéran et Istanbul, et grâce à nos longues observations sur les lieux ainsi quà des entretiens approfondis avec des agents touristiques 
et des personnes ayant effectué récemment un ou plusieurs voyages en Turquie, nous avons cherché à mieux comprendre les enjeux de ces mouvements ${ }^{3}$. Que signifie ce voyage pour les touristes iraniens? Quelles sont les motivations qui poussent les Iraniens à franchir si souvent la frontière entre l'Iran et la Turquie ? En quoi ces simples voyages touristiques renforcent-ils les politiques mercantilistes et favorisent-ils l'essor industriel et commercial de la nation turque?

\section{Les Iraniens en Turquie}

La présence très ancienne des Iraniens à Istanbul connaît son apogée au XIX siècle. Les échanges avec l'Empire ottoman et la Russie ont été à l'origine de l'installation de communautés de négociants à Bakou, à Tiflis, à Batoumi, à Trabzon, à Bursa, à Smyrne, à Alep, à Damas et, bien entendu à Istanbul4. Les Tabrizi (habitants de Tabriz) occupaient alors une place prépondérante, qu'ils fussent chiites ou arméniens. Ensuite, sont venues les migrations plus récentes des Iraniens en Turquie après la révolution iranienne de $1979^{5}$. Selon Jean-François Pérouse, une bonne partie des Iraniens d'Istanbul sont de langue azérie et peuvent, de ce fait, intégrer plus facilement le marché du travail local, du fait de l'absence d'obstacle linguistique ${ }^{6}$. Certains estiment qu'un million d'Iraniens vivent à Istanbul. Pour la majorité, Istanbul - et la Turquie en général - est une étape vers l'Amérique du Nord. Pour beaucoup, cette étape a duré.

Depuis quelques années, les déplacements des Iraniens consistent en voyages ponctuels, soit pour affaires, soit pour le plaisir, et ceux-ci ont pris une telle ampleur qu'ils exigent une observation attentive. Jusqu'à ces dernières années, les touristes iraniens étaient essentiellement originaires des classes moyenne et supérieure de la société. Les couches populaires, elles, se déplaçaient surtout vers les lieux de pèlerinage chiites, les destinations principales à l'étranger étant La Mecque et le Zeynabieh à Damas (avant la guerre en Syrie). Il était fréquent que des tours opérateurs incluent sur le chemin du retour du pèlerinage à Damas une journée de visite et de shopping à Istanbul, un "bonus" pour attirer plus de clients. Mais, depuis 2014, avec le changement de gouvernement et les promotions des tours opérateurs, ces voyages attirent une population plus modeste. Avec les hausses de prix en Iran dues en partie aux sanctions économiques internationales, les vols et séjours tout compris (all included) coûtent parfois moins cher qu'un séjour touristique sur le territoire iranien. La baisse relative du prix des devises depuis 2014 a contribué à l'augmentation de ces mouvements.

\section{Un instrument d'émancipation féminine}

Un aspect important qui caractérise ces voyages est la féminisation des touristes. De manière générale, nous pouvons observer depuis une dizaine d'années une progression des voyages effectués par des femmes - seules, en famille ou entre amies. Sachant que, traditionnellement, les femmes musulmanes ne voyageaient pas seules ${ }^{7}$, ce phénomène pourrait être interprété comme un changement social profond et une avancée significative du statut des femmes, qualifiée de "révolution silencieuse $^{8 ”}$ au sein des sociétés musulmanes du Moyen-Orient.

Aujourd'hui, en dehors de la sphère domestique et des devoirs familiaux, les Iraniennes voyagent

3. L'observation participante nous a aidés à analyser les trajectoires et les enjeux des voyages depuis l'intérieur des groupes. Nous avons mené une trentaine d'entretiens approfondis. 4. Thierry Zarcone, Fariba Zarinebaf, Les Iraniens d'Istanbul, Paris, IFRI, 1993. 5. Fariba Adelkhah, Les Milles et une frontières de I'Iran, Paris, Karthala, 2012. 6. Jean-François Pérouse, "Migrations, circulations et mobilités internationales à Istanbul", in Les dossiers de l'IFEA, $\mathrm{n}^{\circ}$ 13, décembre 2002. 7. Certaines versions des hadiths mentionnent une interdiction inconditionnelle, sans mention aucune d'une quelconque distance. D'autres disent : "Il n'est pas permis à une femme qui croit en Dieu et au jour dernier de voyager la distance de 1 jour et 1 nuit sans qu'elle soit accompagnée d'un homme proche." Dans d'autres versions, il y a "la distance de 2 jours", dans d'autres "Ia distance de 3 nuits" (rapporté par Abû 'Awâna, cité dans Fath ul-bârî, tome 4). 8. Nilufer Göle, Musulmanes et modernes, voile et civilisation en Turquie, Paris, La Découverte, 2003. 
de plus en plus pour leur plaisir et dans leurs déplacements combinent à la fois la consommation et le désir de découverte. Des entretiens avec des femmes voyageant à l'intérieur du pays ou à l'étranger montrent que la plupart visent l'épanouissement personnel mais justifient leur absence de la maison par des raisons économiques (activités commerciales) ou religieuses (pèlerinage). Les voyages dans des lieux "hors du temps", comme l'évoquait Baudelaire à propos de la flânerie, deviennent presque pour les femmes un acte subversif. Ils renversent à leur façon les rapports classiques entre hommes et femmes.

La plupart des femmes enquêtées sont issues des classes moyenne et supérieure avec un niveau d'étude assez élevé, mais certaines sont également issues d'un milieu plus modeste. Elles n'ont, pour la plupart, pas d'emploi fixe en Iran et font du commerce pour avoir une autonomie financière et pour compléter les revenus de la famille. Elles voyagent par petits groupes en passant par des agences de voyages ou en s'organisant entre elles. En général, ces voyages se décident lors des réunions d'anciennes camarades de classe, les jalasseh (cérémonies religieuses effectuées à domicile entre femmes et à l'échelle du quartier) ou pendant les réunions familiales.

À l'opposé des touristes de certains pays de la région qui voyagent avec des tenues spécifiques de leur culture, les Iraniennes restent discrètes et plus difficilement repérables. Anonymes dans la masse, elles sont habillées sobrement et n'ont aucun signe ostentatoire religieux ni ethnique. On peut toutefois les reconnaître, en dehors de leur langue, par l'aspect de leurs cheveux épais ayant subi un brushing soigné ou, pour celles qui gardent le hijab, par la façon particulière de le porter. On voit le plus souvent dépasser les cheveux sous le foulard, à l'inverse d'autres musulmanes non iraniennes dont la tête est strictement couverte. De manière générale, ce sont surtout celles qui viennent en voyage organisé qui conservent leur hijab. Les autres se voient libres de le garder ou de l'enlever.

\section{La Turquie, à la fois si proche et si lointaine}

Les entretiens avec des touristes iraniens soulignent l'importance des valeurs communes entre les deux pays : la croyance et la proximité culturelle (la nourriture, la langue, proche du turc azeri, les coutumes, etc.). Mais ce qui ressort davantage de ces témoignages est le fait de voir dans ce pays voisin un Iran imaginaire, plus "mondialisé" et ouvert sur l'Occident. Ce que l'Iran aurait pu devenir s'il n'avait pas connu de révolution islamique, de guerre et de sanctions de la part

de la communauté internationale. "La Turquie d'aujourd'hui ressemblerait à ce que l'Iran aurait pu devenir si on n'avait pas eu une si grande rupture politique", nous disent avec regret la plupart des Le voyage en Turquie peut aussi être considéré comme un voyage dans le futur. Un modèle de développement pour les jeunes et pour les hommes d'affaires. sur place. Cette déclaration cacherait un sentiment à la fois d'amertume et d'envie envers un pays en plein essor économique qui était, il y a trente ans, moins développé que son voisin iranien. Le voyage en Turquie peut aussi être considéré comme un voyage dans le futur. Un modèle de développement pour les jeunes et pour les hommes d'affaires. Si l'Iran est resté à l'écart du processus de mondialisation qui a fortement touché la Turquie, la proximité géographique et l'effet de contagion ont favorisé une circulation de modèles et de normes qui influent notamment sur les modes de vie et les nouvelles formes de consommation. Les demandes de visas auprès des consulats des pays occidentaux non représentés en Iran tels que les USA et récemment le Canada sont l'un des principaux buts des touristes iraniens. De nombreux tours opérateurs proposent par annonces dans les journaux iraniens, pour une somme forfaitaire de 2000 à 3000 euros, un package comprenant le vol, l'hébergement, le guide et l'obtention d'un 
rendez-vous avec le consulat du pays concerné. La Turquie est également une plaque tournante pour la circulation de devises, dont la sortie est limitée en Iran depuis les sanctions économiques. De nombreux commerçants iraniens ouvrent des comptes en apportant leur argent dans une valise et effectuent leurs transactions avec d'autres pays par l'intermédiaire des banques turques. Cela multiplie les déplacements vers la Turquie en dehors des relations commerciales "officielles" entre les deux pays.

L'investissement dans l'immobilier est un autre motif de voyage des Iraniens. En Turquie, les prix de l'immobilier sont relativement bas comparés aux pays européens. Les prix abordables, sa proximité avec l'Europe et son climat doux font du pays une destination de choix à la fois pour les touristes et pour les expatriés. En Turquie, on n'a pas de mal à trouver des biens adaptés à son budget. Des chambres à 70000 euros ou une luxueuse villa avec vue sur mer pour 1500000 euros.

Les intermédiaires persanophones accueillent les Iraniens pour leur faire visiter des appartements témoins avec vue ou des immeubles réhabilités (dont une partie par les entrepreneurs iraniens) dans le centre d'Istanbul. Les publicités mettent en avant la facilité d'achat de ces biens. Il suffit de payer une partie et le reste sera remboursé par cré- 
dit. Par exemple, en passant par des agences intermédiaires, on peut acheter un appartement de 120000 euros en versant 35000 euros à la signature et demander un crédit pour le complément à une banque turque. Les constructeurs iraniens participent directement au marché de l'immobilier à Istanbul et dans d'autres villes turques. Cette participation reste non officielle et attire les compatriotes sur la base du bouche-à-oreille.

\section{Un lieu propice aux retrouvailles}

Pour attirer et fidéliser la clientèle, les professionnels turcs du tourisme ont tout mis en œuvre. Non contents de mettre à la disposition des touristes les objets de leur convoitise, des biens de consommation exportables, ils jouent sur d'autres besoins plus spécifiques à consommer sur place. En dehors des classiques stations balnéaires, ils proposent des concerts d'artistes iraniens qui ne peuvent se produire dans leur propre pays ou encore, véritables maitres de cérémonie, ils organisent des mariages dans des salles spécialisées. Salles équipées, organisation bien étudiée selon les demandes des familles des mariés et prix compétitifs font que de plus en plus de futurs mariés habitant en Iran célèbrent leur mariage à Istanbul. Les proches sont invités tous frais payés et les autres assument le coût du vol et de l'hébergement. Le mariage est prestigieux, immortalisé à travers de nombreuses photos et des films qui de retour au pays feront des envieux et de nouveaux adeptes. "Nous avons célébré le mariage de ma nièce à Istanbul. La famille du mari vit en Europe et plusieurs membres de sa famille sont mariés à des étrangers. C'était diffcile pour eux de venir en Iran pour assister au mariage. Il fallait des visas, etc. C'était plus simple de l'organiser à Istanbul et on avait entendu que cela se passait très bien là-bas. Tout est prévu et facile. C'est un peu cher la location mais, au bout du compte, c'est plus rentable car on n'est pas obligé d'inviter 200 personnes.
On envoie des cartons d'invitation en Iran à tous nos réseaux en leur annonçant que la fête sera à Istanbul. Certains viennent mais cela ne dépasse pas 40 ou 50 personnes au total. On passe par une agence sur place pour l'hébergement et les repas pendant le séjour des invités. Chacun paie sa part. On passe un moment merveilleux et la fête est magnifique. Ce qui est bien, c'est qu'en Turquie on n'est pas obligé de séparer les hommes des femmes et, au niveau des tenues, on peut s'habiller comme on veut", nous raconte Faézeh, professeur d'université.

La Turquie permet aux membres d'une famille élargie, provenant de différents pays de résidence ou d'exil, de se réunir. Tous se rendent à Istanbul et se retrouvent dans un hôtel ou un appartement loué pour l'occasion. Ils restent en règle générale une semaine à Istanbul et une autre dans une ville côtière, de préférence à Antalya. Shahnaz, une mère de famille cinquantenaire, explique l'intérêt de ces réunions hors frontières : "Toute la famille est dispersée et on se retrouve une fois par an à Istanbul ou ailleurs pour une occasion. L'année dernière, on a fêté deux anniversaires à Istanbul. Mon frère, sa femme, son fils et sa belle-fille sont venus des États-Unis, ma tante et sa fille, ma mère, mon mari et
La Turquie permet aux membres d'une famille élargie, provenant de différents pays de résidence ou d'exil, de se réunir. Tous se rendent à Istanbul et se retrouvent dans un hôtel ou un appartement loué pour l'occasion. moi-même sommes arrivés de Téhéran, et ma fille nous a rejoints depuis Londres. On a passé quatre jours à Istanbul dans un hôtel proche d'Aya Soufia. Nous avons visité des lieux touristiques comme le tombeau de Shah Soltan Hosseyn, le palais Dolma Baghcheh, l'ancienne tour d'Istanbul à vue panoramique où il y a des écritures persanes et même des poèmes de notre poète iranien Hafez. Elle a été construite il y a six cents ans. Puis nous sommes allées à Antalya dans un grand appartement. On s'est amusé, on s'est baigné, on a fait des petits achats et visité les monuments historiques. C'était vraiment un voyage formidable et on veut le faire chaque année si Dieu le veut." 


\section{De l'aventure initiatique au contrôle des flux vers Istanbul}

En Iran, les récits des voyageurs et les séries télévisées turques viennent renforcer cet appel au voyage en Turquie. Les annonces de voyages organisés en Turquie, notamment à Istanbul, abondent dans les journaux iraniens. Nombreuses sont les voyageuses avides de biens de consommation et désireuses d'expérimenter une vie meilleure qui répondent à l'appel et franchissent la frontière pour se détendre loin des interdits imposés par le régime. Se dépayser et se distraire tout en faisant un peu de commerce pour rentabiliser le voyage. Sara, une jeune femme de 28 ans, a déjà voyagé plusieurs fois à Istanbul. Au départ, elle restait entre Iraniens et le guide décidait de tout: "Nous avions peur de quitter le groupe.

Ce commerce informel est

géré essentiellement par

les femmes sur toute la filière depuis l'achat jusqu'à

la distribution. Elles se rendent chaque mois ou tous

les deux mois à Istanbul dans leurs magasins habituels. veulent seulement faire du shopping et voir, à la rigueur, Topkapi ou Soleymanieh. D'autres ne cherchent qu'à s'amuser dans les boîtes de nuit et dans les bars. Mais Istanbul a d'autres aspects qu'il faut découvrir." Ces voyages fréquents entre Téhéran et Istanbul sont formateurs : ils aiguisent la curiosité à l'égard du monde, induisent des comportements nouveaux. Ils permettent aux Iraniens et surtout aux jeunes de découvrir un univers différent et d'expérimenter la dimension internationale sans avoir à quitter définitivement leur pays. Istanbul a su s'adapter habilement à leur demande en leur offrant des possibilités qu'ils ne trouvent pas chez eux tout en se sentant dans un pays musulman et culturellement proche.

Mais, depuis plusieurs années, les gestionnaires du grand Istanbul cherchent à isoler ce type de tourisme des autres en contrôlant les "territoires circulatoires ${ }^{9 "}$ et en créant des espaces artificiels destinés à accueillir les touristes-commerçants à l'extérieur de la ville. Les centres commerciaux comme le Viaport construit près de l'aéroport Sabiha Gökçen où les Iraniens et les Moyen-Orientaux se rendent directement sans avoir même à faire un pas dans Istanbul, les salles de mariage équipées et les hôtels et services aux alentours de ces salles créent peu à peu des archipels vers lesquels volent les touristes iraniens et moyen-orientaux, sans avoir la chance de découvrir cette ville et de se familiariser avec ses différentes facettes. Cette spécialisation des territoires remplace le "voyage" d'aventure en une simple mobilité pendulaire et mécanique entre la ville de résidence et des lieux spécifiques du grand Istanbul, véritables ghettos touristiques où l'ouverture au monde présupposé par le concept même du voyage devient enfermement.

\section{Des voyages aux objectifs multiples}

Comme le rappelle cette expression iranienne courante "ham fâlo ham tamâsha", qui veut dire "tester sa chance et prendre du bon temps", et dont l'expression la plus proche en français serait "avoir le beurre et l'argent du beurre", un voyage doit procurer un plaisir double. Le voyage en Turquie a peu ou prou une double fonction, à la fois touristique et commerciale. Parallèlement à toutes les motivations citées plus haut, la raison principale des voyages des Iraniens à Istanbul reste largement le shopping ou le commerce de marchandises turques devenues extrêmement cotées à Téhéran : l'achat de l'or, réputé de bonne qualité, l'électroménager, les produits en cuir et surtout le prêt-à-porter ${ }^{10}$. 
Selon le rapport de l'Organisation de coopération et de développement économiques $\left(\mathrm{OCDE}^{11}\right)$, la Turquie figure parmi les dix premiers pays exportateurs de vêtements. Depuis 1980 et les programmes de libéralisation économique, la confection représente entre 10 et $20 \%$ des exports et ne cesse d'augmenter. Il existe, en effet, à Istanbul des filières de confection situées hors de l'export officiel. Elles sont associées au commerce à la valise - aussi connu sous le nom de "trabendo" - et alimentent les marchés de consommation qui ne sont pas (encore) dominés par la grande distribution. "Export et commerce à la valise, grande distribution et commerce de détail : à Istanbul, ces deux types de filières d'approvisionnement internationales se sont développés de façon concomitante au sein d'un véritable district industriel urbain dédié à la confection : il représente le premier secteur d'emploi de la métropole (un tiers de la main-d'ceuvre, soit entre 1,5 et 2 millions de personnes) et jusquà $80 \%$ de la production nationale ${ }^{12 . "}$

Cette pratique très courante concerne autant le commerce légal de vente par les boutiques spécialisées dans les produits turcs que le commerce informel effectué dans la plupart des cas par les femmes. Elles sont de plus en plus nombreuses à voyager régulièrement depuis Téhéran, mais aussi depuis les villes secondaires de Shiraz, Tabriz ou Mashad, 
afin de rapporter des marchandises pour leur clientèle féminine (vêtements, chaussures, accessoires, cosmétiques, sous-vêtements). Le commerce informel s'appuie sur un réseau relationnel consolidé et devient une source de l'économie familiale de la classe moyenne urbaine. Ce commerce informel est géré essentiellement par les femmes sur toute la filière depuis l'achat jusqu'à la distribution. Elles se rendent chaque mois ou tous les deux mois à Istanbul dans leurs maga-
Les touristes iraniens,

colporteurs massifs de

marchandises, contribuent

largement au commerce transfrontalier. sins habituels. Les lieux de shopping varient selon le standing de leur clientèle. Cela peut aller des vêtements bon marché jusqu'aux produits de marques. Elles se rendent dans le quartier de Taksim notamment sur l'avenue Istiqlal, vitrine des grandes enseignes donnant une idée de la mode et des prix, puis se dirigent dans les "shopping centers" comme Cevahir ou pour les plus riches Kanyon, un centre commercial à l'américaine très luxueux. Les achats se font également dans les magasins prestigieux des rues de Nisantasi.

Lors de nos dernières observations en juin 2015 au centre commercial Cevahir à Sisli, nous avons observé les deux types de visiteurs iraniens : les flâneurs, ceux qui se promènent en famille et rentrent dans toutes les boutiques pour regarder et acheter des petits cadeaux et souvenirs, des soghati ${ }^{13}$, et les connaisseurs, ceux qui se dirigent directement vers les boutiques qui les intéressent. Ce sont surtout des revendeuses "professionnelles" connaissant le goût et les besoins de leur clientèle en Iran. Les boutiques de marques turques sont situées en face de l'escalator central, très facilement repérable. Par contre, les marques internationales sont placées plus loin, au fond des coursives. Par exemple, les boutiques de prêt-à-porter turques sont sur le chemin des boutiques Zara, Gap etc. Les gestionnaires du centre savent que les Iraniens recherchent systématiquement ces marques, considérées en Iran comme des produits de luxe (à défaut des grandes comme Dior ou Channel).

\section{La visibilité des produits turcs}

L'exposition des produits turcs est largement privilégiée. Il s'agit de prêt-à-porter et accessoires "à la mode", vus à la télévision et portés par les actrices des séries turques, qui se transforment en quelque sorte en mannequins de défilé de mode sans hijab, ce qui est interdit dans la République islamique. Par exemple, les habits et la coiffure de Farkhondeh, personnage joué par Denis Çakir dans le feuilleton célèbre Bargrizan diffusé actuellement tous les soirs sur Gem TV (une télévision iranienne officiellement interdite en Iran), sont vécus comme de véritables modèles pour les femmes en Iran.

Les articles ramenés à Téhéran sont vendus soit dans des boutiques spécialisées lorsqu'il s'agit de commerce formel, soit chez des particuliers lorsqu'il s'agit de commerce informel. En général, ce type de commerce se fait par les femmes pour les femmes. L'exposition-vente se fera chez celle qui a le plus vaste appartement. Dans le parking d'un grand ensemble situé dans les quartiers aisés de Téhéran, des petites annonces indiquent "vente à domicile de vêtements et de produits cosmétiques importés directement de Turquie, appartement X chez madame...". Si l'annonce disparaît assez vite, l'information circule déjà parmi les voisines. Selon l'une des habitantes, plusieurs femmes organisent des ventes de produits turcs dans cet immeuble. "Quand on voit leurs volets fermés, on sait qu'elles sont parties à Istanbul pour rapporter des marchandises. Elles rentrent au bout de quelques temps et leurs appartements se remplissent de monde pendant quelques jours."

Les publicités vantent la provenance turque des produits, qui bénéficie désormais d'un prestige particulier. Souri, la quarantaine, dit : "Avant, les produits turcs n'intéressaient personne en Iran et on cherchait des produits français, etc. Mais aujourd'hui, quand on parle de chaussures ou de vêtements turcs, cela sonne tout de suite plus chic! C'est très différent 
des produits chinois ou indiens qui sont bas de gamme." Cependant, certaines personnes, plus critiques, soupçonnent la Turquie de réserver des produits de moins bonne qualité aux acheteurs iraniens à cause de leur faible pouvoir d'achat.

\section{Un commerce florissant}

Malgré les relations économiques officielles limitées entre les deux pays dues aux sanctions internationales sur l'Iran, nous pouvons constater que les transferts d'argent et de marchandises sont abondants entre les deux pays. Cependant, ces flux restent inégaux car ce sont essentiellement les produits turcs qui sont acheminés vers l'Iran. Les transports de marchandises sont effectués de manière souterraine et informelle par la masse des voyageurs.

Les touristes iraniens, colporteurs massifs de marchandises, contribuent largement au commerce transfrontalier. En réalité, ces "fourmis voyageuses ${ }^{14 "}$, par leurs allers-retours fréquents, contribuent de façon très importante à distribuer des marques européennes et turques. Pour les grands distributeurs dits "fast fashion" comme H\&M, Zara ou Mango, qui renouvellent entièrement leurs collections toutes les 3 semaines, le commerce à la valise est une véritable soupape qui permet d'écouler les surplus de l'export et fait le succès du district industriel ${ }^{15}$.
Les commerçants à la valise achètent en plus petite quantité, mais ils sont nombreux et payent généralement plus cher à l'unité que les grands distributeurs, avec qui les prix sont toujours négociés au plus bas. Ces surplus supplémentaires génèrent davantage de revenus à l'échelle d'Istanbul que l'export à destination de l'Europe. Les revenus dégagés par cette distribution se manifestent par une multiplication du nombre d'intermédiaires entre une production "en miettes" et sa commercialisation $^{16}$.

Grâce aux "gagne-peu ${ }^{17 "}$ qui se déplacent depuis des villes d'Iran pour ramener des marchandises et organiser la revente dans leur réseau de connaissances, les produits voyagent chaque année par millions de valises entre la Turquie et l'Iran. Cela assure la survie de nombreux intermédiaires qui se transforment en agents d'exportation "sans salaire" pour les grands fabricants. Au final, ces derniers ne paieront aucun frais de marketing, de transport, de stockage et d'assurance pour cette distribution massive. Ce sont les "fourmis voyageuses" qui se chargent à leurs risques et périls de la gestion des flux des marchandises importées. Désormais, avec la levée progressive des sanctions envers l'Iran et son ouverture sur le monde, nous pouvons nous demander si ces mouvements vont perdurer avec la même intensité entre ces deux pays. Quelles seront les nouvelles destinations de voyage pour les Iraniens à la recherche d'autres opportunités? 